\title{
Ro-vibrational excitation of CS by He
}

\author{
F. Lique and A. Spielfiedel
}

\author{
LERMA and UMR 8112 of CNRS, Observatoire de Paris-Meudon, 92195 Meudon Cedex, France \\ e-mail: francois.lique@obspm.fr
}

Received 20 September 2006 / Accepted 12 October 2006

\begin{abstract}
Context. Over the next few years, ALMA and Herschel missions will perform high spatial and spectral resolution studies at infrared and sub-millimeter wavelengths. Modeling of the spectra will require accurate radiative and collisional rates for species of astrophysical interest.

Aims. The present paper focuses on the calculation of ro-vibrational excitation rate coefficients of CS by He, useful for studies of high-temperature interstellar environments.

Methods. A new accurate three dimensional (3D) potential energy surface for the CS-He system, which explicitly takes into account the $r$-dependence of the CS vibration, was calculated. The dynamic calculations were performed using the VCC-IOS approximation. Results. Cross sections among the 38 first rotational levels of $v=0, v=1$ and $v=2$ are calculated for total energies up to $10000 \mathrm{~cm}^{-1}$. Rate coefficients between the ro-vibrational levels are calculated for temperatures from $300 \mathrm{~K}$ to $1500 \mathrm{~K}$.
\end{abstract}

Key words. ISM: molecules - molecular data - molecular processes

\section{Introduction}

Observations of molecular emission at millimeter and infrared wavelengths are powerful tools to investigate the physical and chemical conditions of astrophysical objects. Over the last two decades, several diatomic molecules were found in vibrationally excited states in interstellar gas (Turner 1987a,b; Schilke et al. 1992; Cernicharo et al. 1996). These molecules are of interest since they are good probes of extreme physical conditions (near star forming regions, circumstellar envelopes, shocked regions...). Such species can be excited by a strong IR radiation field but also by collisions with other species, in which case they trace regions of very high temperatures and densities (Ziurys \& Turner 1986). Among the vibrationally excited molecules observed, the CS molecule is one of the most frequently observed (Turner 1987a; Walker et al. 1987; Highberger et al. 2000). It plays an important role in astro-chemistry because it is one of the most abundant sulphur-bearing molecules in interstellar clouds. The modeling of CS molecular emission requires excitation calculations using radiative as well as collisional rate coefficients with the most abundant interstellar species: $\mathrm{He}, \mathrm{H}$ and $\mathrm{H}_{2}$. It is thus of importance to provide accurate collisional rate coefficients for the CS molecule for a large temperature range, and for high ro-vibrational levels.

As far as we know, no ro-vibrational excitation rate coefficients exist for the CS molecule. Calculations for the rotational excitation by para- $\mathrm{H}_{2}(j=0)$ were performed by Turner et al. (1992) for the 21 first rotational levels of CS and for temperatures up to $300 \mathrm{~K}$ using a less accurate $\mathrm{CS}-\mathrm{H}_{2}$ potential which was adapted from an electron gas model for the CS-He interaction. Recently, rotational excitation rate coefficients for the $\mathrm{CS}$-He system, based on an accurate ab initio potential energy surface (PES) calculated at the "Coupled Clusters" level and using large atomic basis sets, were determined for temperature ranging from 10 to $300 \mathrm{~K}$ (Lique et al. 2006a). A full CloseCoupling approach was used for the dynamical calculations.
As it involves pure rotational excitation, the CS $r$-distance was kept at its experimental equilibrium geometry for the calculation of the PES. In this paper, we focus on calculations of rate coefficients for the ro-vibrational excitation $(v=0,1$ and 2$)$ of CS by He for temperatures ranging from 300 to $1500 \mathrm{~K}$.

The potential energy surface was calculated for different CS $r$-distances in order to account for the vibration of CS. The same strategy of calculation of the surface for each CS $r$-distance as in Lique et al. (2006a, hereafter Paper I) was adopted here. A full Close-Coupling approach for the dynamics calculations is prohibitively expensive in computer time for heavy molecules when transitions among highly excited ro-vibrational levels are involved. We therefore used the vibrational Close-Coupling rotational Infinite Order Sudden (VCC-IOS) method (Parker \& Pack 1978; Goldflam et al. 1977a,b) to perform the calculations. Cross-sections among the 38 first rotational levels of $v=0$, $v=1$ and $v=2$ have been calculated for total energies up to $10000 \mathrm{~cm}^{-1}$ which give, after Boltzmann thermal average, rate coefficients up to $1500 \mathrm{~K}$.

The paper is organized as follows: Sect. 2 describes the ab initio calculation of the potential energy surface. Section 3 provides a rapid description of the theory and of the calculations. In Sect. 4 we present and discuss our results.

\section{Potential energy surface and computation of matrix elements}

The ground electronic state of the CS-He van der Waals system is a ${ }^{1} \mathrm{~A}^{\prime}$ state. The present work uses a Jacobi coordinate system, in which $r$ is the CS distance, $R$ is the distance from the center of mass of CS to the He atom and $\theta$ is the angle between the two distance vectors $\left(\theta=0^{\circ}\right.$ : He adjacent to carbon atom, $\theta=180^{\circ}$ : He adjacent to sulfur atom). The potential energy surface (PES) was calculated in the supermolecular approach based on the single and double excitation coupled 
cluster method (Hampel et al. 1993) (CCSD) with perturbative contributions of connected triple excitations computed as defined by Watts et al. (1993) (CCSD(T)). The ab initio surface was calculated using the MOLPRO package 2002. The three atoms were described by the standard correlation consistent polarized valence quadruple zeta set of Dunning (1989) (cc-pVQZ) augmented with the diffuse functions of $s, p, d$, f and $g$ symmetries by Kendall et al. (1992) (aug-cc-pVQZ). This basis set is further augmented by the $[3 \mathrm{~s} 3 \mathrm{p} 2 \mathrm{~d} 2 \mathrm{f} 1 \mathrm{~g}]$ bond functions optimized by Cybulski \& Toczylowski (1999) and placed at mid-distance between the He atom and the CS center of mass. The basis set superposition error (BSSE) is corrected at all geometries with the Boys \& Bernardi (1970) counterpoise procedure:

$V(r, R, \theta)=E_{\mathrm{CS}-\mathrm{He}}(r, R, \theta)-E_{\mathrm{CS}}(r, R, \theta)-E_{\mathrm{He}}(r, R, \theta)$

where the CS and He subsystems are computed with the CS-He basis set.

For a van der Waals system, where the ground state is well described by a predominant configuration at all computed geometries, this level of theory is expected to yield reliable results. Calculations were performed for three CS $r$-distances $(2.4,2.9$ and 3.4 bohr) and the same grid of $R$ and $\theta$ values ( $R$ was assigned values from 4.0 bohr to 16.0 bohr by steps of $0.25 \mathrm{bohr}$, the angular grid was uniform with a 15 degrees spacing from 0 to 180 degree) as described in Paper I. A few additional geometries, such that, at any computed angle, the interaction energies becomes at least higher than $10000 \mathrm{~cm}^{-1}$, were computed at shorter internuclear distances $(R \leq 4.0 \mathrm{bohr})$ in order to describe the repulsive part of the potential. A total of 1896 geometries was computed to describe the PES.

The fitting procedure described by Werner et al. (1988) for the $\mathrm{CN}-\mathrm{He}$ system was adopted in order to obtain the $V(r, R, \theta)$ numerical expansion routine required to perform the dynamical calculations. The potential was fitted to the functional form:

$V(r, R, \theta)=\sum_{n=1}^{N_{\max }} \sum_{l=1}^{L_{\max }} d_{m, 0}^{l+m-1}(\cos \theta) A_{l n}(R)\left(r-r_{\mathrm{e}}\right)^{n-1}$

where the $d_{m, 0}^{l+m-1}(\cos \theta)$ are reduced Wigner rotation matrix elements.

$N_{\max }$ equals the number of CS bond distances and $L_{\max }$ the number of angles $\theta$ for which the potential was calculated.

The analytic potential is found to reproduce the calculated surface quite well. Deviations between the fitted potential values and the ab initio points are concentrated in the repulsive part of the PES. Over the entire grid, the mean difference between the analytic fit and the ab initio calculations is $1.5 \%$. In order to check the validity of the interpolation in $r$ of the analytic potential, additional geometries at intermediate $r$-distances were also computed and compared to the fitted potential values: a good agreement (less than 3-4\% of difference) between these values was also found.

For a VCC-IOS calculation, matrix elements of the potential between the vibrational states of the CS molecule are required for fixed values of the Jacobi scattering angle and for all the $R$-values. We write these matrix elements as:

$V_{v, v^{\prime}}(R, \theta)=\left\langle v(r)|V(r, R, \theta)| v^{\prime}(r)\right\rangle$.

We have evaluated the CS vibrational wave functions by the Fourier Grid Hamiltonian (FGH) method of Clay Marston \& Balint-Kurti (1989) from a CS potential calculated with a RKR program (Le Roy 2004). The rotational and vibrational
Table 1. Vibrational matrix elements $\left(\left\langle v^{\prime}(r)\left|\left(r-r_{\mathrm{e}}\right)^{n-1}\right| v^{\prime \prime}(r)\right\rangle\right)$ used in the vibrational averaging of the interaction potential; $r_{\mathrm{e}}$ was taken to be 2.9 bohr.

\begin{tabular}{rc|crr}
\hline \hline$v$ & $v^{\prime}$ & $n=1$ & \multicolumn{1}{c}{$n=2$} & \multicolumn{1}{c}{$n=3$} \\
\hline 0 & 0 & 1.00000 & 0.00827 & 0.00547 \\
0 & 1 & 0.00000 & 0.07344 & 0.00200 \\
0 & 2 & 0.00000 & 0.00379 & -0.00750 \\
1 & 0 & 0.00000 & 0.07367 & 0.00200 \\
1 & 1 & 1.00000 & 0.02445 & 0.01687 \\
1 & 2 & 0.00000 & -0.10412 & -0.00566 \\
2 & 0 & 0.00000 & -0.00750 & 0.00379 \\
2 & 1 & 0.00000 & -0.10444 & -0.00566 \\
2 & 2 & 1.00000 & 0.04081 & 0.02895 \\
\hline
\end{tabular}

constant of the ${ }^{12} \mathrm{C}^{32} \mathrm{~S}$ molecule used in this work were the experimental spectroscopic constants of Bogey et al. (1982). The vibrational wave functions were taken with $j=0$. A very small step size (0.0004 bohr) was used in order to obtain accurate matrix elements.

The relevant $\left\langle v^{\prime}(r)\left|\left(r-r_{\mathrm{e}}\right)^{n-1}\right| v^{\prime \prime}(r)\right\rangle$ matrix elements are given in Table 1.

Contour plots of the $V_{0,0}(R, \theta), V_{0,1}(R, \theta), V_{0,2}(R, \theta)$ and $V_{1,2}(R, \theta)$ surfaces are shown in Fig. 1. In these plots the value $\theta=180^{\circ}$ corresponds to colinear He-SC. The $V_{1,1}(R, \theta) \quad V_{2,2}(R, \theta)$ surfaces are indistinguishable from the $V_{0,0}(R, \theta)$ surface.

The $V_{0,0}(R, \theta)$ matrix element displays the same features as the 2D CS-He PES presented in Paper I. The global minimum in the $V_{0,0}$ surface is found to be $-21.76 \mathrm{~cm}^{-1}(R=7.25 \mathrm{bohr}, \theta=$ $75^{\circ}$ ). We can notice the small anisotropy of the $V_{0,0}(R, \theta)$ matrix elements. The other matrix elements are small compared to the diagonal matrix elements and show, as expected, that the coupling of the $v$ and $v^{\prime}$ levels grows as the scattering coordinate decreases.

\section{The collision problem}

The ground electronic state of the CS molecule is a ${ }^{1} \Sigma$ state. The energies of the first rotational levels belonging to the $v=0$, $v=1$ and $v=2$ vibrational levels are given in Table 2 . They were computed with the use of the experimental spectroscopic constants of Bogey et al. (1982).

In the VCC-IOS method, the rotational levels are treated as being degenerate. Within this approximation the problem reduces to the computation of vibrationally inelastic S-matrix elements calculated with a Close-Coupling approach at fixed $\theta$ Jacobi angles for a given $L$-value. These fixed-angle S-matrix elements must then be multiplied by the appropriate spherical harmonics and integrated over $\theta$ to give the "fundamental IOS cross sections" $\sigma^{\mathrm{IOS}}\left(v, 0 \rightarrow v^{\prime}, L\right)(E)$ out of the $v, j=0$ level.

The de-excitation ro-vibrational cross sections are expressed in a reduced form in terms of the $\sigma^{\mathrm{IOS}}\left(v, 0 \rightarrow v^{\prime}, L\right)$ cross sections (Parker \& Pack 1978):

$\sigma^{\mathrm{IOS}}\left(v, j \rightarrow v^{\prime}, j^{\prime}\right)=\sum_{L}\left(2 j^{\prime}+1\right)\left(\begin{array}{ccc}j^{\prime} & j & L \\ 0 & 0 & 0\end{array}\right)^{2} \sigma^{\mathrm{IOS}}\left(v, 0 \rightarrow v^{\prime}, L\right)$

where ( ) is the " $3-j$ " symbols.

$v, j$ and $v^{\prime}, j^{\prime}$ are respectively the initial and final vibrational and rotational states. The summation in Eq. (4) was performed for $L \leq 75$ which allows us to obtain converged cross sections for rotational angular momenta $j \leq 37$. All the calculations were performed using the MOLSCAT computer program 

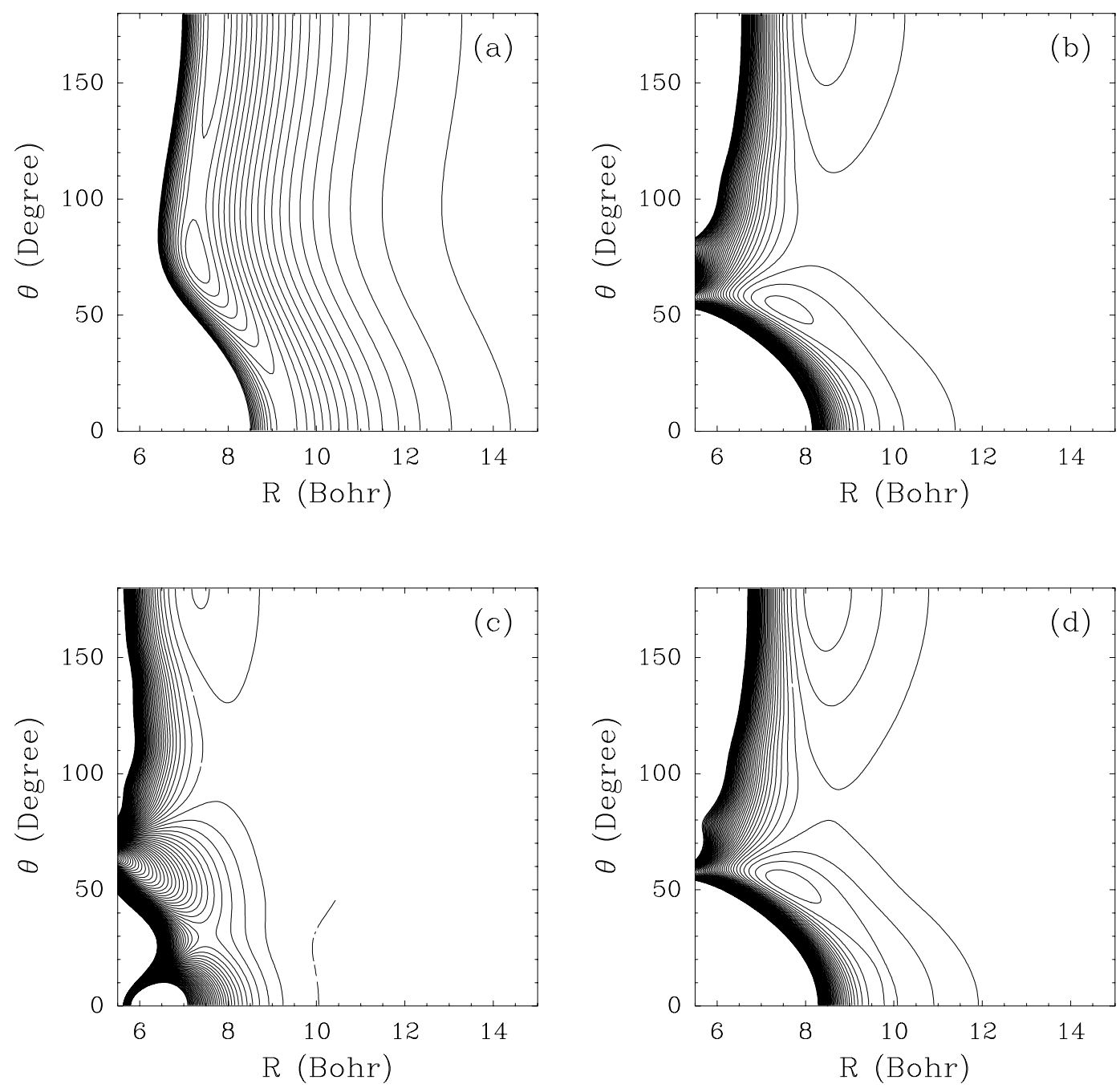

Fig. 1. Contour plots of the $V_{0,0}(R, \theta)$ a), $\left.\left.V_{0,1}(R, \theta) \mathbf{b}\right), V_{0,2}(R, \theta) \mathbf{c}\right)$ and $\left.V_{1,2}(R, \theta) \mathbf{d}\right)$ surfaces. The energies are in $\mathrm{cm}^{-1}$ with a distance of $1.0 \mathrm{~cm}^{-1}$ between two adjacent contours for the a) plot, with a distance of $0.1 \mathrm{~cm}^{-1}$ between two adjacent contours for the b) and d) plot, and with a distance of $0.02 \mathrm{~cm}^{-1}$ between two adjacent contours for the c) plot, the zero of energy is taken as that of the He+CS asymptote.

(Hutson \& Green 1995). The calculations were carried out using the propagator of Manolopoulos (1986). The reduced mass of the system is $3.669 \mathrm{amu}$. Typically, the minimum and maximum integration distances are respectively $R_{\min }=3.5 a_{0}$ and $R_{\max }=$ $40 a_{0}$.

Rate coefficients are obtained by averaging the appropriate cross sections over a Boltzmann distribution of velocities at a given kinetic temperature $T$ :

$$
\begin{aligned}
k_{v, j \rightarrow v^{\prime}, j^{\prime}}= & \left(\frac{8 \beta^{3}}{\pi \mu}\right)^{1 / 2} \int_{0}^{\infty} \sigma\left(v, j \rightarrow v^{\prime}, j^{\prime}\right)\left(E_{k}\right) \\
& \times E_{k} \exp \left(-\beta E_{k}\right) \mathrm{d} E_{k}
\end{aligned}
$$

where $\beta=\left(k_{\mathrm{B}} T\right)^{-1}, k_{\mathrm{B}}$ is the Boltzmann constant, $\mu$ is the reduced mass of the colliding system. The total energy $E$ is related to the kinetic energy according to $E=E_{k}+\epsilon_{v, j}$ where $\epsilon_{v, j}$ is the energy of the initial rovibrational level. Rate coefficients for the reverse transitions may be obtained by detailed balance.

As CS is a heavy molecule with a small rotational constant, the IOS approach, although it neglects the energy structure of the rotational levels and is expected to be poor at low energies, seems to be appropriate for the temperature range considered in this work $(T>300 \mathrm{~K})$. However it may not be so appropriate for vibrational quenching as resonant vibrational energy transfer may occur. This type of near-resonant energy transfer has been observed experimentally (McCaffery et al. 2000; Stewart et al. 1988) and obtained theoretically (Stewart et al. 1988, 2000; Krems et al. 2001) for diatomic molecules with a small moment of inertia $\left(\mathrm{H}_{2}, \mathrm{HF}, \mathrm{Li}_{2}\right)$. Molecules with smaller rotational constants (CO for example) can exhibit resonant vibrational relaxation when they are initially in very high rotational levels. The role of near-resonant vibrational relaxation has been investigated systematically in CO (Krems 2002). This study shows that the contribution of this resonant process is negligible for low $j$ levels and high energy, and becomes only important when the initial rotational state of $v=1$ (or 2) is close in energy to high rotational states of $v=0$ (or 1) and when the $\Delta j$ of the transition is small. Such a case is not considered here since we have not included in the calculation rotational levels of $v=0$ higher in energy than the first rotational levels of $v=1$ and rotational levels of $v=1$ higher in energy than the first rotational levels of $v=2$.

\section{Results}

In order to compute cross sections and rate coefficients for vibrational relaxation of CS, we have tested the convergence of the vibrational Close-Coupling expansion by including several vibrational wave functions in the basis set. 
Table 2. Energy in $\mathrm{cm}^{-1}$ of the rotational levels of CS considered in this work.

\begin{tabular}{|c|c|c|c|c|c|c|c|c|c|c|c|}
\hline Level & $v$ & $j$ & Energy & Level & $v$ & $j$ & Energy & Level & $v$ & $j$ & Energy \\
\hline 1 & 0 & 0 & 0.000 & 39 & 1 & 0 & 1172.151 & 77 & 2 & 0 & 2331.299 \\
\hline 2 & 0 & 1 & 1.634 & 40 & 1 & 1 & 1173.773 & 78 & 2 & 1 & 2332.910 \\
\hline 3 & 0 & 2 & 4.902 & 41 & 1 & 2 & 1177.018 & 79 & 2 & 2 & 2336.131 \\
\hline 4 & 0 & 3 & 9.805 & 42 & 1 & 3 & 1181.884 & 80 & 2 & 3 & 2340.962 \\
\hline 5 & 0 & 4 & 16.341 & 43 & 1 & 4 & 1188.373 & 81 & 2 & 4 & 2347.403 \\
\hline 6 & 0 & 5 & 24.511 & 44 & 1 & 5 & 1196.484 & 82 & 2 & 5 & 2355.455 \\
\hline 7 & 0 & 6 & 34.315 & 45 & 1 & 6 & 1206.217 & 83 & 2 & 6 & 2365.117 \\
\hline 8 & 0 & 7 & 45.752 & 46 & 1 & 7 & 1217.571 & 84 & 2 & 7 & 2376.388 \\
\hline 9 & 0 & 8 & 58.823 & 47 & 1 & 8 & 1230.547 & 85 & 2 & 8 & 2389.269 \\
\hline 10 & 0 & 9 & 73.526 & 48 & 1 & 9 & 1245.144 & 86 & 2 & 9 & 2403.759 \\
\hline 11 & 0 & 10 & 89.862 & 49 & 1 & 10 & 1261.362 & 87 & 2 & 10 & 2419.859 \\
\hline 12 & 0 & 11 & 107.831 & 50 & 1 & 11 & 1279.200 & 88 & 2 & 11 & 2437.567 \\
\hline 13 & 0 & 12 & 127.431 & 51 & 1 & 12 & 1298.658 & 89 & 2 & 12 & 2456.883 \\
\hline 14 & 0 & 13 & 148.663 & 52 & 1 & 13 & 1319.736 & 90 & 2 & 13 & 2477.807 \\
\hline 15 & 0 & 14 & 171.526 & 53 & 1 & 14 & 1342.433 & 91 & 2 & 14 & 2500.338 \\
\hline 16 & 0 & 15 & 196.020 & 54 & 1 & 15 & 1366.749 & 92 & 2 & 15 & 2524.476 \\
\hline 17 & 0 & 16 & 222.144 & 55 & 1 & 16 & 1392.683 & 93 & 2 & 16 & 2550.221 \\
\hline 18 & 0 & 17 & 249.897 & 56 & 1 & 17 & 1420.235 & 94 & 2 & 17 & 2577.572 \\
\hline 19 & 0 & 18 & 279.279 & 57 & 1 & 18 & 1449.404 & 95 & 2 & 18 & 2606.528 \\
\hline 20 & 0 & 19 & 310.290 & 58 & 1 & 19 & 1480.190 & 96 & 2 & 19 & 2637.088 \\
\hline 21 & 0 & 20 & 342.929 & 59 & 1 & 20 & 1512.592 & 97 & 2 & 20 & 2669.253 \\
\hline 22 & 0 & 21 & 377.194 & 60 & 1 & 21 & 1546.609 & 98 & 2 & 21 & 2703.021 \\
\hline 23 & 0 & 22 & 413.087 & 61 & 1 & 22 & 1582.240 & 99 & 2 & 22 & 2738.392 \\
\hline 24 & 0 & 23 & 450.604 & 62 & 1 & 23 & 1619.486 & 100 & 2 & 23 & 2775.365 \\
\hline 25 & 0 & 24 & 489.747 & 63 & 1 & 24 & 1658.344 & 101 & 2 & 24 & 2813.939 \\
\hline 26 & 0 & 25 & 530.514 & 64 & 1 & 25 & 1698.815 & 102 & 2 & 25 & 2854.114 \\
\hline 27 & 0 & 26 & 572.904 & 65 & 1 & 26 & 1740.897 & 103 & 2 & 26 & 2895.888 \\
\hline 28 & 0 & 27 & 616.916 & 66 & 1 & 27 & 1784.589 & 104 & 2 & 27 & 2939.261 \\
\hline 29 & 0 & 28 & 662.550 & 67 & 1 & 28 & 1829.892 & 105 & 2 & 28 & 2984.231 \\
\hline 30 & 0 & 29 & 709.804 & 68 & 1 & 29 & 1876.802 & 106 & 2 & 29 & 3030.799 \\
\hline 31 & 0 & 30 & 758.678 & 69 & 1 & 30 & 1925.321 & 107 & 2 & 30 & 3078.962 \\
\hline 32 & 0 & 31 & 809.171 & 70 & 1 & 31 & 1975.446 & 108 & 2 & 31 & 3128.720 \\
\hline 33 & 0 & 32 & 861.281 & 71 & 1 & 32 & 2027.177 & 109 & 2 & 32 & 3180.072 \\
\hline 34 & 0 & 33 & 915.007 & 72 & 1 & 33 & 2080.513 & 110 & 2 & 33 & 3233.016 \\
\hline 35 & 0 & 34 & 970.349 & 73 & 1 & 34 & 2135.452 & 111 & 2 & 34 & 3287.552 \\
\hline 36 & 0 & 35 & 1027.304 & 74 & 1 & 35 & 2191.993 & 112 & 2 & 35 & 3343.679 \\
\hline 37 & 0 & 36 & 1085.873 & 75 & 1 & 36 & 2250.135 & 113 & 2 & 36 & 3401.395 \\
\hline 38 & 0 & 37 & 1146.054 & 76 & 1 & 37 & 2309.878 & 114 & 2 & 37 & 3460.699 \\
\hline
\end{tabular}

We have first considered the effect of vibrational coupling on the rotational excitation cross sections. Figure 2 compares the fundamental IOS cross sections (see Eq. (4)) for vibrationally elastic rotational excitation of CS out of the $v=0, j=0$ level calculated with and without the vibrational coupling between $v=$ 0 and $v=1$.

As already found for other systems like CO (Krems 2002) and HF (Krems et al. 2001; Krems \& Nordholm 2001), the pure rotational transitions are unaffected by the vibrational channel included in the calculation. This result is directly the consequence of the very small non-diagonal vibrational matrix elements of the interaction potential compared to the diagonal ones. In addition, we found an excellent agreement between the cross sections calculated with the 3D PES presented here and the one calculated with the 2D PES (Lique 2006). This result, already found for the SO molecule in collisions with $\mathrm{He}$ (Lique et al. 2006 b), validates the choice of a 2D PES for pure rotational excitation.

We have also considered the convergence of the vibrational expansion on inelastic vibrational cross sections. Table 3 shows the "fundamental IOS cross sections" out of $v=1, j=0$ (see Eq. (4)) for vibrational $v=0-1, v=0-2$ and $v=0-3$ basis sets.

For the range of considered collision energies $\left(0-10000 \mathrm{~cm}^{-1}\right)$, this table clearly shows that the values of the

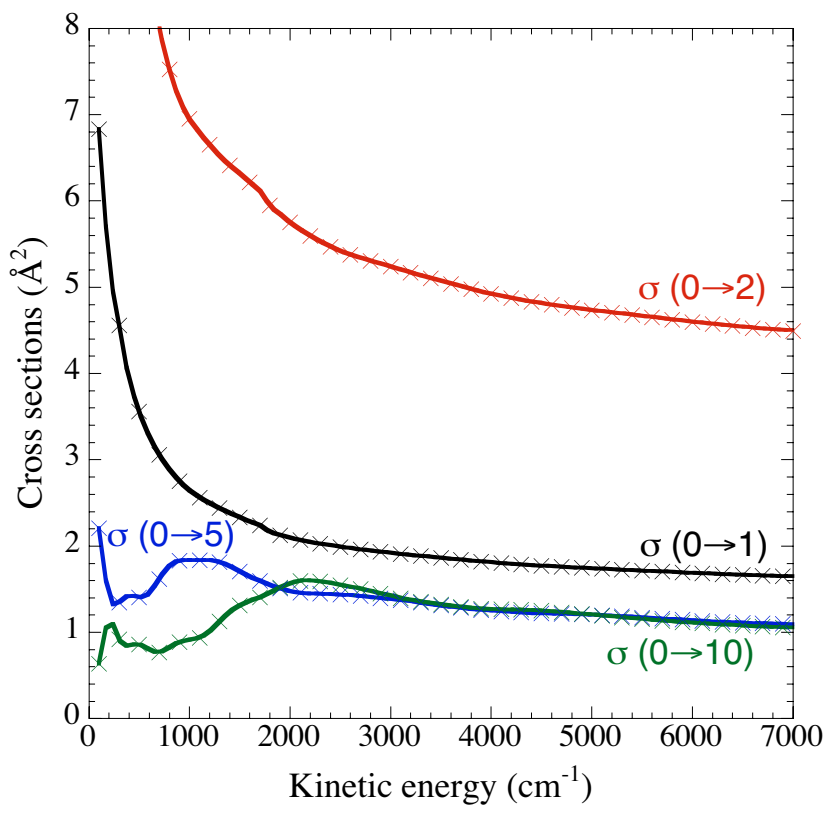

Fig. 2. Variation of the vibrationally elastic rotational cross sections for $v=0$ with the $v=0$ and $v=1$ basis set (full curve) and without vibrational coupling (crosses). 
Table 3. "Fundamental IOS cross sections" $\sigma\left(v, 0 \rightarrow v^{\prime}, L\right)\left(\AA^{2}\right)$ (see Eq. (4)) for vibrationally elastic rotational excitation of CS out of the $v=1, j=0$ level to $v=0, L$ level calculated including 2,3 and 4 vibrational levels in the dynamical calculations.

\begin{tabular}{|c|c|c|c|c|c|c|}
\hline & Basis set & $\sigma(1,0 \rightarrow 0,1)$ & $\sigma(1,0 \rightarrow 0,2)$ & $\sigma(1,0 \rightarrow 0,5)$ & $\sigma(1,0 \rightarrow 0,10)$ & $\sigma(1,0 \rightarrow 0,50)$ \\
\hline \multicolumn{7}{|l|}{$E=1500 \mathrm{~cm}^{-1}$} \\
\hline & $v=0-1$ & $1.17 \times 10^{-7}$ & $2.06 \times 10^{-7}$ & $4.67 \times 10^{-7}$ & $1.95 \times 10^{-7}$ & $2.68 \times 10^{-11}$ \\
\hline & $v=0-2$ & $1.24 \times 10^{-7}$ & $2.20 \times 10^{-7}$ & $5.13 \times 10^{-7}$ & $2.23 \times 10^{-7}$ & $2.65 \times 10^{-11}$ \\
\hline & $v=0-3$ & $1.24 \times 10^{-7}$ & $2.20 \times 10^{-7}$ & $5.13 \times 10^{-7}$ & $2.23 \times 10^{-7}$ & $2.65 \times 10^{-11}$ \\
\hline \multicolumn{7}{|l|}{$E=2500 \mathrm{~cm}^{-1}$} \\
\hline & $v=0-1$ & $4.83 \times 10^{-6}$ & $1.88 \times 10^{-5}$ & $1.99 \times 10^{-5}$ & $2.78 \times 10^{-5}$ & $7.92 \times 10^{-7}$ \\
\hline & $v=0-2$ & $5.09 \times 10^{-6}$ & $2.06 \times 10^{-5}$ & $2.11 \times 10^{-5}$ & $3.05 \times 10^{-5}$ & $8.47 \times 10^{-7}$ \\
\hline & $v=0-3$ & $5.09 \times 10^{-6}$ & $2.06 \times 10^{-5}$ & $2.11 \times 10^{-5}$ & $3.05 \times 10^{-5}$ & $8.47 \times 10^{-7}$ \\
\hline \multicolumn{7}{|l|}{$E=5000 \mathrm{~cm}^{-1}$} \\
\hline & $v=0-1$ & $8.28 \times 10^{-5}$ & $3.50 \times 10^{-4}$ & $2.78 \times 10^{-4}$ & $5.26 \times 10^{-4}$ & $1.49 \times 10^{-4}$ \\
\hline & $v=0-2$ & $9.63 \times 10^{-5}$ & $3.83 \times 10^{-4}$ & $3.25 \times 10^{-4}$ & $6.45 \times 10^{-4}$ & $2.10 \times 10^{-4}$ \\
\hline & $v=0-3$ & $9.68 \times 10^{-5}$ & $3.84 \times 10^{-4}$ & $3.26 \times 10^{-4}$ & $6.50 \times 10^{-4}$ & $2.13 \times 10^{-4}$ \\
\hline
\end{tabular}
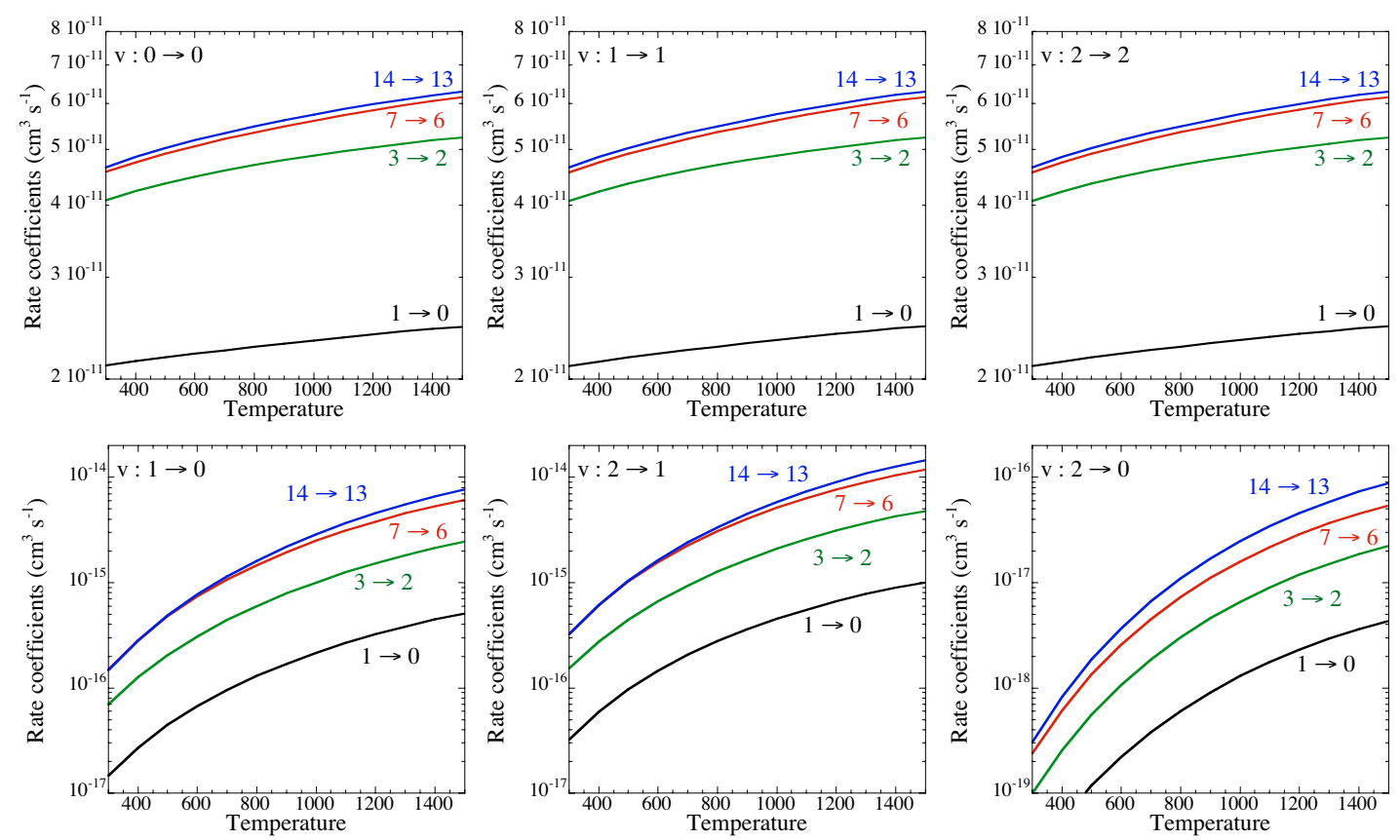

Fig. 3. Temperature variation of the $k_{v, j \rightarrow v^{\prime}, j^{\prime}}$ collision rates for vibrationally elastic transitions (upper panel) and vibrationally inelastic ones (lower panel).

cross sections converge to better than one per cent on including one closed vibrational channel in the coupled equations. Therefore, all the calculations presented in the following part were performed with the $v=0-3$ basis set.

Calculations were performed for the 38 first rotational levels of $v=0, v=1$ and $v=2$ for energies up to $10000 \mathrm{~cm}^{-1}$ leading to converged rate coefficients for all considered levels and temperatures up to $1500 \mathrm{~K}$. The temperature variation of the rate coefficients involving the first rotational levels (rate coefficients corresponding to transitions of astrophysical interest) are displayed in Fig. 3 for $v=0 \rightarrow v^{\prime}=0, v=1 \rightarrow v^{\prime}=1$, $v=2 \rightarrow v^{\prime}=2, v=1 \rightarrow v^{\prime}=0, v=2 \rightarrow v^{\prime}=1$ and $v=2 \rightarrow v^{\prime}=0$ transitions.

One observes a slow variation with temperature of the vibrationally elastic rate coefficients when the variation of the vibrational excitation $v=1 \rightarrow v=0, v=2 \rightarrow v=1$ and $v=2 \rightarrow v=0$ coefficients vary more rapidly. As found for other systems, the rate coefficients for $\Delta v=1,2$ transitions are lower than the elastic coefficients by several orders of magnitude even at relatively high temperature. It would be necessary to consider much higher temperatures and/or high- $j$ levels leading to near-resonant $j$-levels belonging to $v=0$ (or 1 ) and $v=$ 1 (or 2) to get much higher vibrational excitation rate coefficients. It is thus expected that the values obtained in the present VCC-IOS approximation for vibrational excitation and de-excitation give the correct order of magnitude of the rate coefficients.

It is also interesting to note a relative independence of the vibrational level to the vibrationally elastic rate coefficients. Indeed, the value of the rate coefficients for given rotational transitions is very similar whatever the $v$ level considered (see Fig. 3 upper panels). Contrarily, the rate coefficients for vibrationally inelastic transition $\Delta v=1$ vary with the initial $v$ level.

These results are pointed out in Table 4 which collects rate coefficients at three different temperatures for vibrational relaxation and purely rotational excitation for several $v, j=0$ states of CS.

Hence, the vibrational energy transfer is enhanced by increasing $v$ whereas rate coefficients for vibrationally elastic total rotational relaxation are almost not affected by a change in the vibrational level. These results, even if they are similar to those obtained for the CO molecule in collision with He (Krems 2002), 
Table 4. Rate coefficients at $T=300,800$ and $1500 \mathrm{~K}$ for total vibrational relaxation and total rotational excitation of CS $(v, j=0)$ by $\mathrm{He}$ $\left(\mathrm{cm}^{3} \mathrm{~s}^{-1}\right)$. The notation $\sum j^{\prime}$ is used for the rate coefficients summed over $j^{\prime}$ levels.

\begin{tabular}{cccc}
\hline \hline$v$ & $T(\mathrm{~K})$ & $k_{v, j=0 \rightarrow v, \Sigma j^{\prime}}$ & $k_{v, j=0 \rightarrow v-1, \Sigma j^{\prime}}$ \\
\hline & 300 & $3.72 \times 10^{-10}$ & $\ldots$ \\
0 & 800 & $6.30 \times 10^{-10}$ & $\ldots$ \\
& 1500 & $8.64 \times 10^{-10}$ & $\ldots$ \\
\hline & 300 & $3.73 \times 10^{-10}$ & $5.57 \times 10^{-16}$ \\
1 & 800 & $6.32 \times 10^{-10}$ & $2.41 \times 10^{-14}$ \\
& 1500 & $8.66 \times 10^{-10}$ & $1.96 \times 10^{-13}$ \\
\hline & 300 & $3.73 \times 10^{-10}$ & $1.23 \times 10^{-15}$ \\
2 & 800 & $6.32 \times 10^{-10}$ & $5.12 \times 10^{-14}$ \\
& 1500 & $8.66 \times 10^{-10}$ & $3.80 \times 10^{-13}$ \\
\hline
\end{tabular}

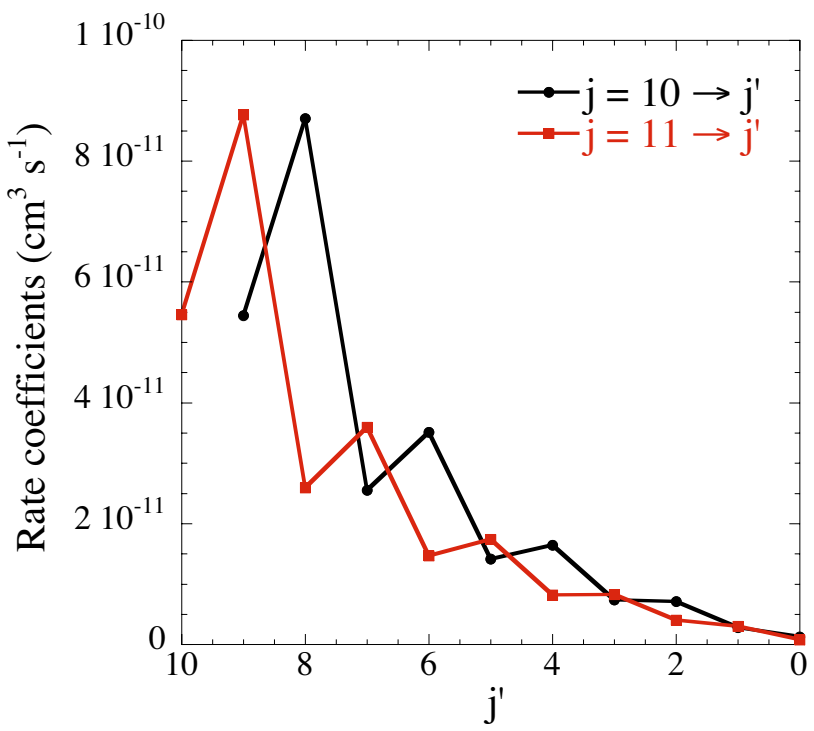

Fig. 4. Downward rate coefficients at $1000 \mathrm{~K}$ from the $v=0, j=10$ and $v=0, j=11$ levels to $v=0, j^{\prime}$ levels.

should be confirmed by a more systematic study taking into account more vibrational levels or by experimental measurements.

We are also interested in the propensity rules of pure rotational transitions. Figure 4 presents downward vibrationally elastic rate coefficients at $1000 \mathrm{~K}$ from the $v=0, j=10$ and $v=0, j=11$ levels to $v=0, j^{\prime}$ levels.

The downward rate coefficients decrease with increasing $\Delta j$, which is the usual trend, and propensity rules exist: the rate coefficients for even $\Delta j$ transitions are larger than the ones for odd $\Delta j$ transitions. The propensity to favor even $\Delta j$ over odd $\Delta j$ was well explained by McCurdy \& Miller (1977) in terms of an interference effect related to the anisotropy of the PES: due to the large even anisotropy of the CS-He PES, the even $\Delta j$ transitions are favored.

Figure 5 presents diagrams of the final rotational state distribution $v^{\prime}=0, j^{\prime}$ after vibrational relaxation of various $v=$ $1, j$ states at a temperature of $1000 \mathrm{~K}$.

The rate coefficients have clear maxima at the final rotational quantum numbers $j^{\prime}$ equal to the initial rotational quantum numbers. More generally, the vibrational relaxation from a $j$ level exhibits a peak centered on the region of low $j^{\prime}$ levels with a strong propensity for the $\Delta j=0$ transitions. As $j^{\prime}$ becomes smaller or larger than $j$, the rate coefficients decrease but display a long tail for high $j^{\prime}$. The shape of the distribution differs when the initial rotational level is small $(j<5)$ as illustrated by Fig. 5 (upper panel) where we can see a distribution peak centered around higher $j^{\prime}$ than the initial $j$. This kind of distribution is similar to the one found by Palov et al. (2002) for the vibrational relaxation of $\mathrm{SiO}$ in collisions with $\mathrm{H}$. However, some caution must be exercised regarding this distribution since the VCC-IOS method used in this work neglects the rotational structure of the molecule and by consequence the possible resonant vibrational relaxation when the initial and final rotational levels are close to each other.

All the rate coefficients are available on our web site http: //www . obspm. fr/basecol/.

\section{Summary and discussion}

In this work, we use the vibrational Close-Coupling - rotational IOS approach to investigate the ro-vibrational energy transfer in collisions of CS with He atoms. The calculations are performed with a new accurate $3 \mathrm{D}$ potential energy surface. The results can be summarized as follows:

(i) The vibrationally elastic rotational energy transfer in $v=0$ is essentially unaffected by the vibrational coupling with the higher vibrational levels. An analogous behaviour was observed by Krems (2002) in their study of vibrational and rotational excitation of $\mathrm{CO}$ by collisions with $\mathrm{He}$. From an analysis of the rotational excitation of $\mathrm{CO}(v, j=0)$ for different $v$-values, these authors conclude that the vibrational motion of HF plays no role in pure rotational transitions. This result, confirmed for other colliding systems like SO with He (Lique et al. 2006b) or HF with Ar (Krems \& Nordholm 2002), is important as cross sections and rate coefficients for rotational excitation may thus be obtained without considering the vibration of the diatomic molecule.

(ii) In the energy range considered $(T \leq 1500 \mathrm{~K})$ the rate coefficients for vibrational excitation are several orders of magnitude lower than the rotational rate coefficients. As a consequence, vibrational excitation of $\mathrm{CS}$ by $\mathrm{He}$ may be considered as negligible in comparison with radiative vibrational excitation in the majority of nonLocal Thermodynamic Equilibrium (LTE) astrophysical environments.

(iii) The calculations performed within the VCC-IOS approximation cannot take into account the near-resonant interactions that may occur between highly excited rotational levels in $v=0,1$ and $v=1,2$. Such an effect is small for the low or moderately high rotational levels and high energies considered in this work. However, a complete study of this system should be done using Close-Coupling or Coupled States approach to confirm the present results.

It is somewhat difficult to assess the absolute accuracy of our calculated rates. We believe that the typical error may be lower than an order of magnitude, and that the results are probably accurate within a factor of 2-3.

In the interstellar medium, the large abundance of $\mathrm{H}_{2}$ makes this species the first colliding partner for the other molecules. It is generally assumed (Schöier et al. 2005) that rate coefficients with $\mathrm{He}$ can provide an estimation of rate coefficients with para$\mathrm{H}_{2}(j=0)$. The underlying approximation is to consider identical cross sections for the two colliding systems and apply a scaling factor to account for the different associated reduced masses. However, some caution must be exercised concerning the use of the present rate coefficients in collisions with He to provide a first estimate of rate coefficients with para- $\mathrm{H}_{2}(j=0)$. Recent results on rotational excitation of CO (Wernli et al. 2006), 

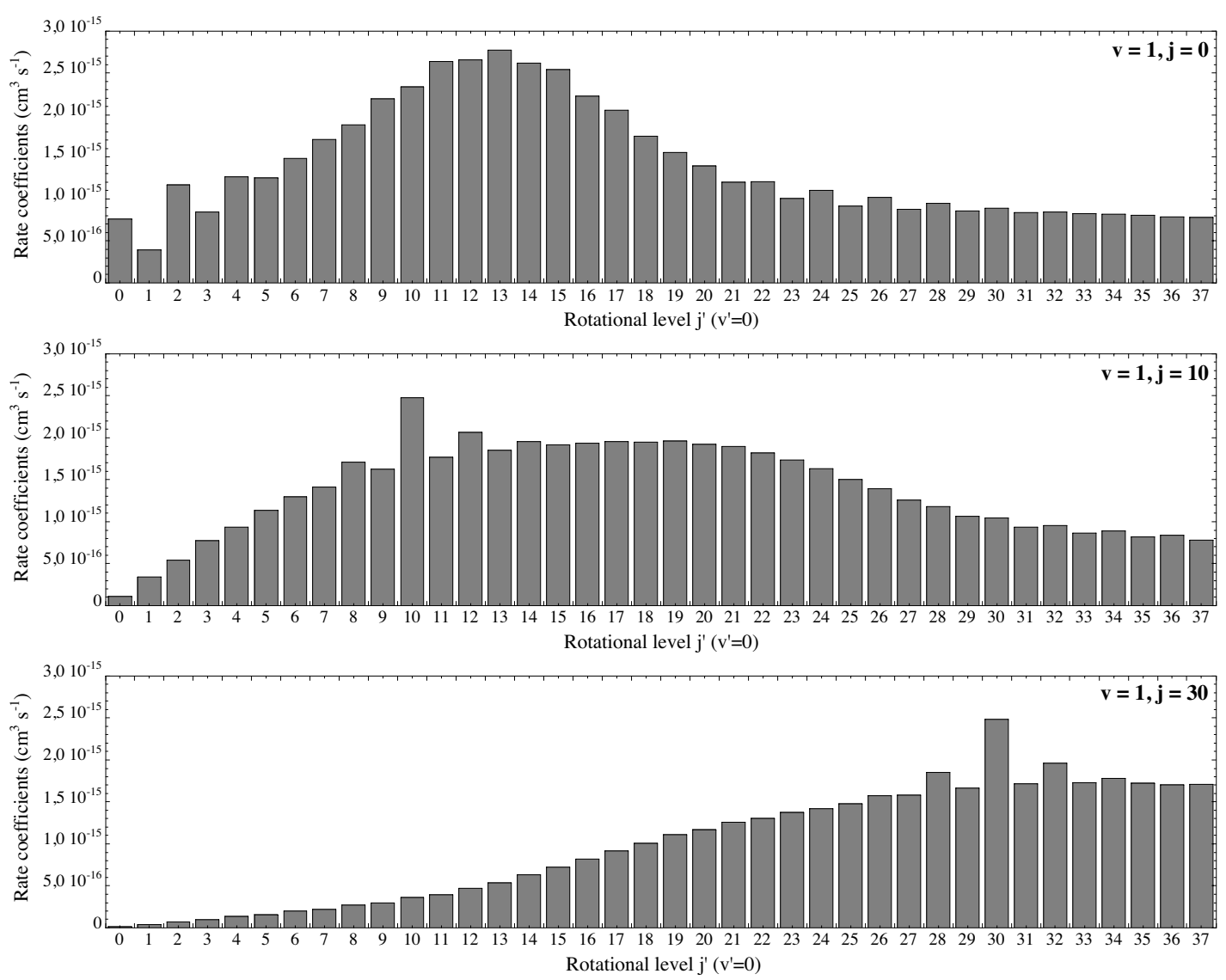

Fig. 5. Distribution of the final rotational levels $j^{\prime}$ in $v^{\prime}=0$ following vibrational relaxation of $v=1, j$ states at $T=1000 \mathrm{~K}$. Upper panel is for $j=0$ level, middle panel is for $j=10$ level and bottom panel is for $j=30$ level.

$\mathrm{SiO}$ (Dayou \& Balança 2006), CS (Lique 2006) pointed out that rate coefficients with para- $\mathrm{H}_{2}(j=0)$ are within a factor $1-3$ larger or lower than those with $\mathrm{He}$, depending on the selected transition. This indicates that accurate rate coefficients for collisions with $\mathrm{H}_{2}$ cannot be obtained from the present results although they give the correct order of magnitude.

Acknowledgements. We would like to acknowledge N. Feautrier and F. Dayou for their continuing interest in this work and fruitful discussions. We also acknowledge G. Dhont for making available to us his fitting code of the potential energy surface. The calculations of ab initio potential energy surfaces were performed on the parallel machine MPOPM of Paris Observatory, and all the scattering calculations were performed at the IDRIS-CNRS center (Institut de Développement et des Ressources en Informatique Scientifique du Centre National de la Recherche Scientifique) under project 050883.

\section{References}

Bogey, M., Demuynck, C., \& Destombes, J.-L. 1982, J. Mol. Spect., 95, 35 Boys, S. F., \& Bernardi, F. 1970, Mol. Phys., 19, 553

Cernicharo, J., Barlow, M. J., Gonzalez-Alfonso, E., et al. 1996, A\&A, 315, L201

Clay Marston, C., \& Balint-Kurti, G. G., 1989, J. Chem. Phys., 91, 3571

Cybulski, S. M., \& Toczylowski, R. R. 1999, J. Chem. Phys., 111, 10520

Dayou, F., \& Balança, C. 2006, A\&A, in press

Dunning, T. H. 1989, J. Chem. Phys., 90, 1007

Goldflam, R., Green, S., \& Kouri, D. J. 1977a, J. Chem. Phys., 67, 4149

Goldflam, R., Kouri, D. J., \& Green, S. 1977b, J. Chem. Phys., 67, 5661

Hampel, C., Peterson, K., \& Werner H.-J. 1992, Chem. Phys. Lett., 190, 1

Highberger, J. L., Apponi, A. J., Bieging J. H., \& Ziurys, L. M. 2000, ApJ, 544, 881

Kendall, R. A., Dunning, T. H., \& Harrison, R. J. 1992, J. Chem. Phys., 96, 6796 Krems, R. V. 2002, J. Chem. Phys., 116, 4517

Krems, R. V., \& Nordholm, S. 2001, J. Chem. Phys., 115, 257
Krems, R. V., Markovic, N., Buchachenko, A. A., \& Nordholm, S. 2001, J. Chem. Phys., 114, 1249

Lique, F. 2006, Thèse: excitation collisionnelle de molécules d'intérêt astrophysique: théorie et interprétations d'observations

Lique, F., Spielfiedel. A., \& Cernicharo, J. 2006a, A\&A, 451, 1125

Lique, F., Spielfiedel, A., Dhont, G., \& Feautrier, N. 2006b, A\&A, 458, 331

Le Roy, R. J. 2004, University of Waterloo Chemical Physics Research Report CP-657R

Manolopoulos, D. E. 1986, J. Chem. Phys., 85, 6425

McCaffery, A. J., \& Marsh, R. J. 2000, J. Phys. Chem., 104, 10442

McCurdy, C. W., \& Miller, W. H. 1977, J. Chem. Phys., 67, 463

MOLPRO is a package of ab initio programs written by:

Werner, H.-J. and Knowles, P. J. with contributions from Almlöf, J., Amos, R. D., Deegan, M. J., Elbert, S. T., Hampel, C., Meyer, W., Peterson, K., Pitzer, R., Stone, A. J., Taylor, P. R., Lindh, R., Mura, M. E., \& Thorsteisson, T.

Hutson, J. M., \& Green, S. 1994, MOLSCAT computer code, version 14, Collaborative Computational Project No. 6 of the Science and Engineering Research Council, UK

Parker, G. A., \& Pack, R. T. 1978, J. Chem. Phys., 68, 1585

Palov, A. P., Jimeno, P., Gray, M. D., Field, D., \& Balint-Kurti, G. G. 2002, J. Chem. Phys., 116, 1338

Schilke, P., Güsten, R., Schulz, A., Serabyn, E., \& Walmsley, C. M. 1992, A\&A, 261, L5

Schöier, F. L., van der Tak, F. F. S., van Dishoeck, E. F., \& Black, J. H. 2005 , A\&A, 432, 369

Stewart, B., Magil, P. D., Scott, T. P., Derouard, J., \& Pritchard, D. E. 1988, Phys. Rev. Lett., 60, 282

Stewart, B., Magil, P. D., \& Pritchard, D. E. 2000, J. Phys. Chem., 104, 10565

Turner, B. E. 1987a, A\&A, 182, L15

Turner, B. E. 1987b, A\&A, 183, L23

Turner, B. E., Chan, K.-W., Green, S., \& Lubowich, D. A. 1992, ApJ, 399, 114

Walker, C. K., Maloney, P. R., \& Serabyn, E. 1994, ApJ, 437, L127

Watts, J. D., Gauss, J., \& Bartlett, R. J. 1993, J. Chem. Phys., 98, 8718

Werner, H.-J., Follmeg, B., \& Alexander, M. H. 1988, J. Chem. Phys., 89, 3139

Wernli, M., Valiron, P., Faure, A., et al. 2006, A\&A, 446, 367

Ziurys, L. M., \& Turner, B. E. 1986, ApJ, 300, L19 